\title{
Diagnóstico Y EVALUACióN DEL TRANSPORTE DE MERCANCÍAS POR FERROCARRIL EN COLOMBIA
}

Juan Diego Pineda-Jaramillo ${ }^{1}$

\author{
${ }^{1}$ M.Sc. en Ingeniería, Infraestructura y Sistemas de Transporte. Estudiante de Doctorado, \\ Departamento de Ingeniería e Infraestructura de los Transportes, Universitat Politècnica de València, España. \\ Correo electrónico: juapija1@doctor.upv.es
}

Recibido: 10 de febrero del 2016 Aprobado: 11 de julio del 2016

Cómo citar este artículo: J. D. Pineda-Jaramillo, "Diagnóstico y evaluación del transporte de mercancías por ferrocarril en Colombia", Ingeniería Solidaria, vol. 12, no. 20, pp. 151-161, oct. 2016. doi: http://dx.doi.org/10.16925/in.v12i20.1480

Resumen. Introducción: este artículo es producto de la investigación desarrollada en la tesis de Maestría titulada "Modelo de elección de modo de transporte entre carretera y ferrocarril para carga general susceptible a exportación-importación. Aplicación al corredor vial Medellín-Puerto de Cartagena”, de la Universidad Nacional de Colombia. El objetivo de la investigación es realizar un diagnóstico del ferrocarril en Colombia para descubrir los motivos por los que la industria colombiana, después de haber utilizado principalmente el ferrocarril para transportar sus mercancías por más de 100 años, no utiliza actualmente este modo de transporte, y así evaluar sus posibilidades futuras para transportar mercancías. Metodología: en este estudio se realizó una investigación cualitativa para analizar la evolución del ferrocarril desde su creación hasta su declive y el estado actual del ferrocarril colombiano para el transporte de mercancías. Luego se desarrolló un grupo focal para conocer los motivos por los que la industria colombiana no utiliza el ferrocarril. Resultados: los motivos son principalmente la falta de infraestructura idónea para realizar el transbordo de la mercancía y de confianza en el modo. Conclusiones: es ingente la necesidad de facilitar la implementación de una integración multimodal para el transporte de mercancías en Colombia; de esta manera, el Gobierno colombiano podrá incidir en las variables que la industria considera importantes para utilizar el ferrocarril al momento de implementar una renovación de los ferrocarriles.

Palabras clave: Colombia, ferrocarril, industria colombiana, transporte de mercancías. 


\title{
Diagnosis and Evaluation of Railway Transport of Goods in Colombia
}

\begin{abstract}
Introduction: This article is the result of research carried out in the master's thesis titled "Road and Railway transport mode choice model for general cargo to be exportedimported. Application to the Medellin-Port of Cartagena road system", by Universidad Nacional de Colombia. The purpose of the research is to develop a diagnosis of the railway system in Colombia to discover the reasons why the Colombian industry, after mainly using the railway system to transport its goods for more than 100 years, is not using this mode of transport at present, to assess future possibilities to use this system for the transport of goods. Methodology: A qualitative research was conducted in this study to analyze the evolution of the railway from its creation to its decline and the current status of the Colombian railway system for the transport of goods. A focus group was then carried out to establish why the Colombian industry does not use the railway. Results: These reasons mainly include the lack of suitable infrastructure for the transfer of goods and the reliability of such mode of transport. Conclusions: The need to facilitate the implementation of a multimodal integration for freight transport in Colombia is huge; thus, the Colombian Government may influence the variables considered relevant for the industry to use the railway system when upgrading the railways.
\end{abstract}

Keywords: Colombia, railway, Colombian industry, transport of goods.

\section{Diagnóstico E AVAliação do TRANSPORTE FERroviárIO dE MERCADORIAS NA COLÔMBIA}

Resumo. Introdução: este artigo é resultado da pesquisa desenvolvida na tese de mestrado titulada "Modelo de eleição de modalidade de transporte entre estrada e ferrovia para carga geral susceptível a exportação-importação. Aplicação ao corredor viário Medellín-Puerto de Cartagena", da Universidade Nacional da Colômbia. O escopo da pesquisa é fazer um diagnóstico do transporte ferroviário na Colômbia para descobrir as motivações da indústria colombiana que, depois de ter utilizado principalmente o transporte ferroviário para transportar suas mercadorias durante mais de 100 anos, não utiliza mais esta modalidade de transporte e avaliar então suas possibilidades futuras para transportar mercadorias. Metodologia: neste estudo foi feita uma pesquisa qualitativa para analisar a evolução do transporte ferroviário desde sua origem até seu declínio e o atual estado do transporte ferroviário colombiano para o transporte de mercadorias. Depois um grupo focal foi desenvolvido para conhecer os motivos pelos quais a indústria colombiana não utiliza o trem. Resultados: esses motivos são principalmente a falta de infraestrutura adequada para realizar o transbordo da mercadoria e de confiança na modalidade. Conclusões: é enorme a necessidade de facilitar a implementação de uma integração multimodal para o transporte de mercadoria na Colômbia; dessa forma, o Governo colombiano poderá incidir nas variáveis consideradas importantes para a indústria para utilizar o trem no momento de implementar uma renovação dos trens.

Palavras chave: Colômbia, transporte ferroviário, indústria colombiana, transporte de mercadorias. 


\section{Introducción}

Muchos historiadores afirman que en Colombia se construyeron los ferrocarriles con el propósito de transportar café a los puertos [1-4], ya que era el producto que más se movilizaba en el país; por ejemplo, desde 1942 hasta 1962 el café representaba más del $80 \%$ de las exportaciones, y en 1985 representaba más del $50 \%$ [5]. Con el pasar de los años, el ferrocarril perdió protagonismo y el transporte de mercancías fue pasándose a las carreteras, se perdieron muchas líneas ferroviarias por falta de uso y en la actualidad no existe un sistema ferroviario eficiente en Colombia, sino unos fragmentos de lo que este debería ser.

Mientras países desarrollados como Estados Unidos, China y Rusia transportaron en el 2014, cada uno, más de dos billones de toneladas-km de mercancías por ferrocarril (lo que demuestra la competitividad de este modo para el transporte de mercancías [6]), Colombia sigue apostando por las carreteras para transportar mercancías, invirtiendo más de 40 billones de pesos en el 2013 en este modo, en el programa Cuarta generación de concesiones [7]. Además, en Colombia el transporte de mercancías por carretera presenta problemas, como altos costos de operación, gran impacto negativo en el medio ambiente, altos índices de accidentalidad en las vías, altos tiempos de viaje, etc. [8].

En el 2013 se transportaron 220000 toneladas de mercancías en $203000 \mathrm{~km}$ de carreteras, mientras que por ferrocarril se transportaron 76000 toneladas de mercancías, de las cuales el 99,9\% fue carbón transportado en 350 km de dos líneas ferroviarias, la administrada por la empresa Drummond y la administrada por la empresa Cerrejón [9]. De esta información se deduce que aunque el transporte ferroviario en Colombia se limita a un solo producto (carbón), este conformó el 25,5\% de la mercancía transportada en el 2013 en una red ferroviaria muchísimo menor que la red de carreteras, hecho que demuestra la gran capacidad del ferrocarril para transportar una amplia magnitud de mercancías; además, es importante subrayar que únicamente el 0,03\% de las mercancías en Colombia (sin incluir carbón) se transporta en ferrocarril, lo que muestra que se sigue basando toda la movilización de la mercancía en un solo modo, y de esta manera se evita el uso del ferrocarril que tiene grandes características técnicas, lo que permitiría complementar otros sistemas de transporte, como el de carretera, y repercutir en la economía del país.

Los factores que tiene en cuenta la industria a nivel mundial para elegir un modo u otro se dividen en cuatro categorías [10]: 1) costos (de manipulación, de transporte, de confiabilidad del servicio, etc.); 2) atributo físico de la mercancía (tamaño, características, valor, densidad, etc.); 3) características de distribución (frecuencia del servicio, distancia del envío, etc.); y 4) características del modo de transporte (capacidad, confiabilidad del viaje y del tiempo de viaje, servicio al cliente, calidad de manejo de la mercancía, etc.). Es razonable deducir que las intervenciones políticas pueden cambiar el balance entre estos factores, dependiendo de las necesidades de la industria de cada país.

Este artículo pretende presentar un recorrido histórico del ferrocarril en Colombia e indagar sobre los motivos por los que la industria no lo utiliza en la actualidad, para poder actuar en ellos y lograr que una reactivación, tan necesaria, del ferrocarril en Colombia sea exitosa.

Este artículo presenta, aparte de esta introducción, un estado del arte, mostrando los primeros años del ferrocarril y su declive, además de presentar el estado actual del ferrocarril colombiano y el transporte de mercancías en Colombia. Posteriormente se presenta la discusión en la cual se describen los motivos por los que la industria no utiliza las pocas líneas ferroviarias en operación que quedan en la actualidad; finalmente, en las conclusiones, se proponen lineamientos que debería considerar el Gobierno colombiano para implementar una renovación completa de los ferrocarriles colombianos, teniendo en cuenta las variables que influyen en la elección de este modo por la industria.

\section{Metodología}

La metodología seguida para realizar un diagnóstico del ferrocarril en Colombia y descubrir los motivos por los cuales la industria colombiana no utiliza este modo de transporte y poder evaluar las posibilidades de este para el transporte de mercancías fue la siguiente:

1. Analizar la historia de lo que fue el ferrocarril colombiano, desde su creación, con el propósito de transportar café a los puertos, hasta su 
declive, al 2016 es utilizado casi en su totalidad por solo dos empresas para transportar carbón.

2. Determinar cuál es el estado actual del ferrocarril colombiano, las líneas en operación, las activas y el material rodante existente.

3. Identificar el reparto modal de las mercancías que son transportadas en Colombia.

4. Realizar un grupo focal que permita conocer los motivos por los que la industria colombiana no utiliza el ferrocarril.

\section{Evolución del ferrocarril colombiano}

\subsection{Primeros años del ferrocarril}

La construcción de ferrocarriles en Colombia comenzó a finales del siglo xIX con el ferrocarril de Panamá, entre los puertos de Colón (sobre el océano Atlántico) y Panamá (sobre el Pacífico) entre 1849 y 1854 [11], cuando Panamá aún era una provincia colombiana.

Después de la construcción del ferrocarril de Panamá, se construyeron muchos otros ferrocarriles, como el de Antioquia, Caldas, Cundinamarca, La Dorada, La Sabana, Tolima (Huila), entre otros, que fueron concebidos con un carácter local, articulándose a los ríos Cauca y Magdalena que estaban en condiciones de navegar en ese momento.

Los ferrocarriles en Colombia siempre fueron aumentando en longitud y capacidad conforme la producción de café iba creciendo, y de igual forma su exportación, pues de 1932 a 1943 se aumentó la producción de café en un $33 \%$ [12]. Este sistema funcionó hasta mediados del siglo $\mathrm{xx}$, cuando se decidió nacionalizar los ferrocarriles y se crearon entonces en 1954 los Ferrocarriles Nacionales de Colombia (FNC) para reunir a todo el sistema ferroviario con el fin de centralizar la operación y el mantenimiento de los ferrocarriles del país que ya estaba un poco dañada.

Pero a causa de la rápida exploración, extensión y construcción que se llevaba en las carreteras, a partir de los años treinta se comenzó un desmantelamiento de los ferrocarriles colombianos, y progresivamente, hasta los años sesenta, se había levantado un gran número de carriles. Paradójicamente, este fue el período cuando más cantidad de vía ferroviaria se alcanzó a construir, con un total de $3462 \mathrm{~km}$, pues el sector privado (Ecopetrol y Cementos Diamante) añadió redes ferroviarias y se logró transportar la mayor cantidad de toneladas en el máximo número de kilómetros posibles. A partir de los años setenta se ve cómo el transporte nacional pasa a las carreteras nacionales sin importar que el ferrocarril cafetero hubiese sido la fórmula para acceder con el café a los mercados internacionales en la primera apertura económica del país [13].

\subsection{El declive del ferrocarril}

A partir de 1975, se hizo evidente en el FNC una aguda crisis financiera originada por problemas de índole laboral, administrativo, técnico, operativo y financiero. Por otra parte, los criterios de explotación de la red no tuvieron en cuenta elementos de rentabilidad, por lo que se operaron líneas improductivas creando subsidios cruzados en la operación. Todo esto, unido a la falta de políticas integrales de transporte y al exceso de funciones que desempeñaba la empresa, incrementó sustancialmente los costos operativos frente a los bajos recaudos por la prestación del servicio. La consecuencia obvia fue un déficit operativo considerable y creciente [14]. En octubre de 1986, tras analizar la situación financiera de la empresa, el Gobierno determinó reexaminar la viabilidad económica del transporte ferroviario [14].

Ante esta situación, la primera tarea consistió en modificar la estructura institucional del ferrocarril, con patrones de eficiencia y rentabilidad. Posteriormente, se creó un fondo de pasivo social para atender las obligaciones prestacionales y en 1991 el Gobierno colombiano decidió liquidar al FNC y crear una nueva entidad llamada Empresa Colombiana de Vías Férreas (Ferrovías) para reemplazar la vieja entidad, y se autorizó la creación de empresas de transporte ferroviario de carácter privado o público-privado, para la explotación y comercialización del sistema [15].

Ferrovías diseñó el proceso de concesión integral y en 1998 creó las contrataciones públicas destinadas a la celebración de los dos grandes contratos del Estado en este campo. Este consistió en la rehabilitación, mantenimiento y operación de la infraestructura ferroviaria. 
Después se otorgó por este sistema la red del Pacífico a Tren de Occidente S. A. en 1998 y la red del Atlántico a Fenoco en 1999 [16].

Dada la incapacidad de cumplir las tareas propuestas por parte del Gobierno a Ferrovías, mediante el Decreto 1791 de 26 de junio de 2003, el Gobierno nacional decretó su liquidación, la cual debía cumplirse, a más tardar, dentro de los dos años siguientes al 26 de junio del 2003, prorrogables en dos años más; a través del Decreto 2386 de 2007. Se amplió el término de la liquidación hasta el 27 de junio del 2008, indicando que, en todo caso, al finalizar el plazo el proceso debía culminar y la empresa debía desaparecer jurídicamente como sujeto de derecho, para todos los efectos [17].

Así, tras su liquidación surgió el documento del Consejo Nacional de Política Económica y Social (CONPES) \# 3581 como un proyecto de importancia estratégica del proyecto "Concesión Red Férrea del Atlántico", a cargo del Ministerio de Transporte y el Instituto Nacional de Concesiones (INCO) que tenía como fin rediseñar y replantear las inversiones a futuro para el ferrocarril y así reactivar este medio de transporte de manera competente en el país, es decir, se transferían las obras adjudicadas al INCO [18].

Posteriormente surgió el ConPEs \# 3655, cuyo fin era la declaratoria de importancia estratégica de la interventoría de la "Red Férrea del Pacífico", así como la modificación al perfil de aportes aprobado mediante el documento Conpes \# 3581 de 2009 para la interventoría de la Red Férrea del Atlántico; ambas interventorías estuvieron a cargo del INCO, que posteriormente se convirtió en la Agencia Nacional de Infraestructura (ANI) [19].

Actualmente, la ANI se ha encargado de realizar algunas proyecciones de lo que podría ser la red ferroviaria del país, con el fin de adjudicar nuevas concesiones y evaluar iniciativas de empresas privadas que vieran provechoso para ellas la reactivación del ferrocarril [20]. Algunos de los proyectos presentados por el Gobierno colombiano son la concesión del ferrocarril central (centro del país al departamento del Cesar), el ferrocarril al Puerto de la Brisa (Guajira), ferrocarril del Carare (Cundinamarca, Boyacá y Santander a puertos del Atlántico) y la concesión del ferrocarril de Boyacá
- Belencito [21]. Esto demuestra que el Gobierno colombiano tiene interés particular en autorizar varios proyectos, mostrando la necesidad de implementar el uso del ferrocarril para el transporte de mercancías. Para conseguir el objetivo mencionado, el Gobierno colombiano estableció la necesidad de involucrar el sector privado; de esta forma, la construcción, rehabilitación, mantenimiento, operación y explotación de la red ferroviaria podría ser posible.

\section{Resultados}

\subsection{Estado actual del ferrocarril colombiano}

Colombia tiene una red ferroviaria con una longitud de más de $3800 \mathrm{~km}$ al 2014 [9], caracterizada por especificaciones técnicas que han sido mantenidas desde su inicio, incluyendo radios pequeños y tangentes cortas que siguen la forma de la topografía de los lugares donde ha sido construida. La mayoría de la red ferroviaria está inactiva.

En cuanto a la red activa, la longitud de las líneas ferroviarias en operación ha disminuido a lo largo de la última década, hasta llegar a tener únicamente $854 \mathrm{~km}$ en el 2014, como se muestra en la tabla 1 [9]. Estas líneas son utilizadas para transportar mercancías, y en algunos casos para transportar pasajeros. Aunque estas líneas han sido restauradas, no han sido completamente renovadas para cumplir con condiciones técnicas de acuerdo con los estándares internacionales.

La red inactiva supone $3017 \mathrm{~km}$ en el 2014, lo que representa el $78 \%$ de la red ferroviaria total de ese mismo año (tabla 1). Esta red, en general, está críticamente dañada, con tramos desprovistos de carriles, traviesas y a veces con balasto casi inexistente; algunos corredores tienen invasiones de población, y existen tramos con poca estabilidad debido a fallas geológicas y otros factores. Cabe destacar la ausencia de información precisa relativa a los tramos de la red inactiva que, en su mayor parte, se encuentran abandonados y en importante estado de degradación [22]. 
Tabla 1. Infraestructura ferroviaria al 2014

\begin{tabular}{|c|c|c|c|c|c|c|c|}
\hline \multirow[b]{2}{*}{ Año } & \multicolumn{3}{|c|}{ Línea ferroviaria en operación } & \multicolumn{4}{|c|}{ Línea ferroviaria inactiva } \\
\hline & Nacional & Privado & Total & $\begin{array}{c}\text { Concesión - } \\
\text { Atlántico }\end{array}$ & $\begin{array}{c}\text { Concesión - } \\
\text { Pacífico }\end{array}$ & Nación & Total \\
\hline 2002 & 1972 & 240 & 2212 & 263 & $\mathrm{ND}^{*}$ & $\mathrm{ND}^{*}$ & 263 \\
\hline 2003 & 1991 & 240 & 2231 & 160 & $\mathrm{ND}^{*}$ & $\mathrm{ND}^{*}$ & 160 \\
\hline 2004 & 1992 & 145 & 2137 & 118 & $\mathrm{ND}^{*}$ & $\mathrm{ND}^{*}$ & 118 \\
\hline 2005 & 1992 & 145 & 2137 & 118 & $\mathrm{ND}^{*}$ & $\mathrm{ND}^{*}$ & 118 \\
\hline 2006 & 1992 & 145 & 2137 & 118 & $\mathrm{ND}^{*}$ & $\mathrm{ND}^{*}$ & 118 \\
\hline 2007 & 1518 & 145 & 1663 & 118 & $\mathrm{ND}^{*}$ & $\mathrm{ND}^{*}$ & 118 \\
\hline 2008 & 1488 & 184 & 1672 & 118 & $\mathrm{ND}^{*}$ & $\mathrm{ND}^{*}$ & 118 \\
\hline 2009 & 1488 & 184 & 1672 & 118 & $\mathrm{ND}^{*}$ & $\mathrm{ND}^{*}$ & 118 \\
\hline 2010 & 1039 & 184 & 1223 & 118 & $\mathrm{ND}^{*}$ & $\mathrm{ND}^{*}$ & 118 \\
\hline 2011 & 1010 & 184 & 1194 & 118 & $\mathrm{ND}^{*}$ & $\mathrm{ND}^{*}$ & 118 \\
\hline 2012 & 756 & 184 & 940 & 118 & $\mathrm{ND}^{*}$ & $\mathrm{ND}^{*}$ & 118 \\
\hline 2013 & 610 & 184 & 794 & 118 & $\mathrm{ND}^{*}$ & 1787 & 1905 \\
\hline 2014 & 670 & 184 & 854 & 118 & 1170 & 1729 & 3017 \\
\hline
\end{tabular}

$\mathrm{ND}^{*}$ : no hay información

Fuente: [9]

Por otro lado, la evolución del material rodante existente en las líneas ferroviarias en operación se muestra en la figura 1. A partir del 2009, se incluyen las locomotoras y los vagones carboneros de Drummond y Cerrejón [23].
Cabe destacar que la red ferroviaria colombiana es una de las pocas que aún operan con ancho de vía angosto $(0,914 \mathrm{~m})$, con excepción de $150 \mathrm{~km}$ de la línea privada de Cerrejón que tiene ancho de vía estándar de $1435 \mathrm{~mm}$ [24], lo que

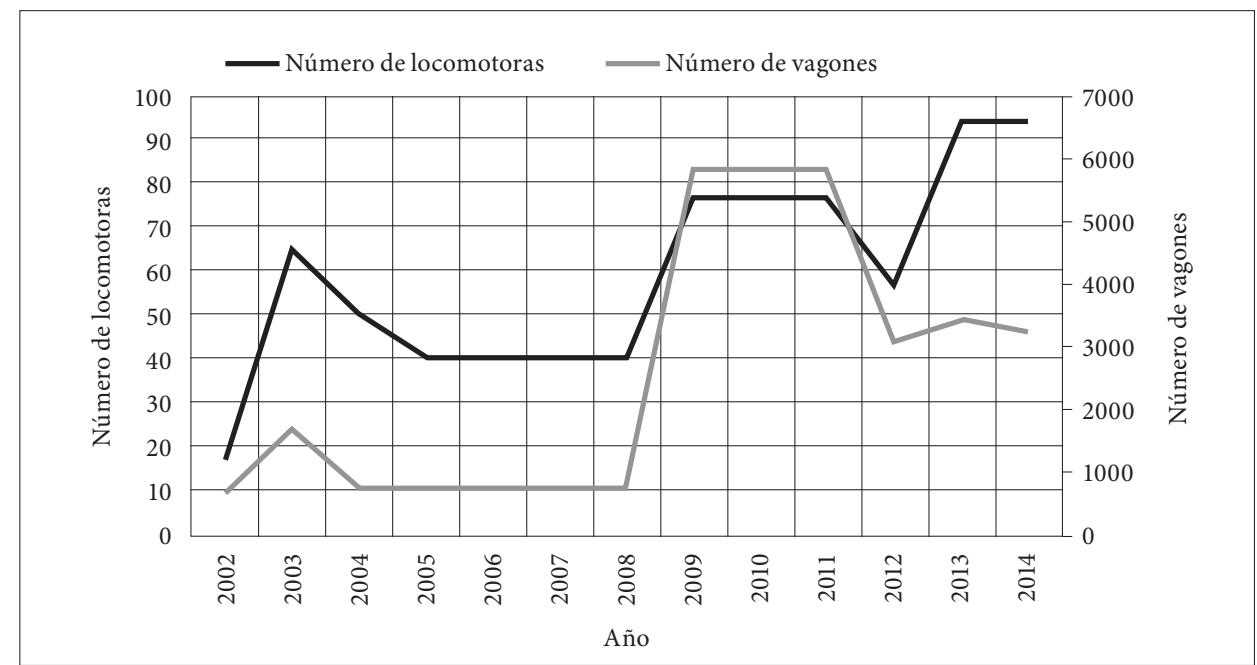

Figura 1. Evolución de unidades de transporte del modo ferroviario colombiano entre 2002 y 2014 Fuente: [9] 
hace que el hecho de encontrar equipo de material rodante sea más difícil que el usado para ancho de vía estándar u otros anchos de uso más comunes a nivel internacional.

\subsection{Transporte de mercancías en Colombia}

La movilización de mercancías de un país indica su nivel de desarrollo, su nivel de productividad y su índice de competitividad. El transporte de mercancías en Colombia, en el 2013, fue de 301 millones de toneladas, de las cuales se destacan 220,3 millones de toneladas transportadas por carretera y 76,8 millones transportadas por ferrocarril (se destaca ampliamente el transporte de carbón con el 99,9\% de las mercancías transportadas por ferrocarril). Las 3,9 millones de toneladas restantes estuvieron repartidas en los modos fluvial, aéreo y cabotaje [9]. La figura 2 muestra el reparto modal de las mercancías transportadas en Colombia al 2013.

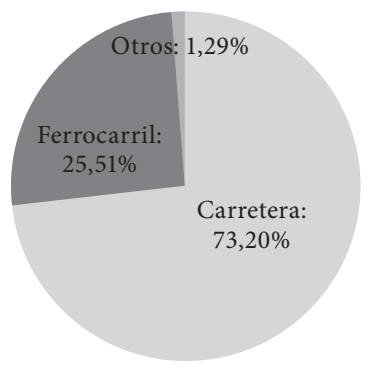

Figura 2. Reparto modal de las mercancías que son transportadas en Colombia

Fuente: elaboración propia con información de [9]
Por otro lado, la figura 3 presenta la evolución del transporte de mercancías por ferrocarril y por carretera para el período 2002-2013 [9].

La figura 3 muestra que las mercancías transportadas en Colombia han seguido una tendencia creciente, y el porcentaje de las mercancías transportadas por ferrocarril se ha mantenido constantes en el período presentado.

Entre las empresas que utilizan el ferrocarril para transportar carbón, se destacan Cerrejón, que transportó 32 millones de toneladas en el 2011, en una línea de $150 \mathrm{~km}$ [25], y Drummond, que por su parte transportó 25 millones de toneladas en el 2012 en una línea de 193 km [26].

\section{3 ¿Por qué la industria colombiana no usa el ferrocarril?}

Un grupo focal se define como una discusión cuidadosamente diseñada para obtener las percepciones sobre una particular área de interés [27]. Estos grupos deben ser lo suficientemente pequeños como para permitir la oportunidad a cada participante de compartir su discernimiento de las cosas, y a la vez lo suficientemente grandes como para proveer diversidad de percepciones; un número adecuado es entre seis y doce participantes [28].

El objetivo del grupo focal era conocer la opinión que tenía la industria, que actualmente transporta sus mercancías por vías diferentes al ferrocarril, sobre el ferrocarril, y saber los criterios que tienen en cuenta para realizar la elección del modo para transportar mercancías.

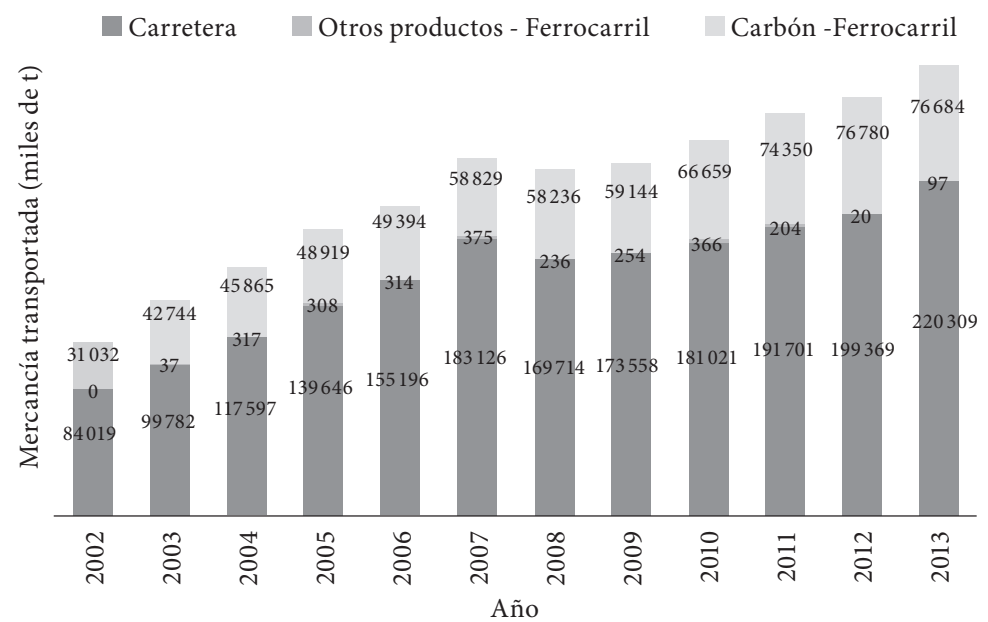

Figura 3. Mercancía transportada por carretera y por ferrocarril en el período 2002-2013 en Colombia (en miles de toneladas) Fuente: elaboración propia con información de [9] 
El grupo focal fue conformado por ocho personas encargadas del área de transporte y comercio exterior capaces de generar una discusión con la cual se pudiera extraer la información necesaria. Los miembros del grupo focal eran de empresas que generaban/atraían grandes volúmenes de mercancías, como Cristalería Peldar, Sofasa, Productos Familia y Omya. El grupo focal fue realizado en abril del 2012. En general, todos los miembros del grupo focal transportan sus mercancías por carretera, porque es el modo más económico del que disponen en la actualidad, ya que evitan realizar transbordos.

En cuanto a los motivos por los que no progresan los proyectos de construcción y operación de ferrocarriles para transportar mercancías en Colombia, los asistentes del grupo focal expresaron su inconformidad con la existencia de infraestructura con fines logísticos mal ubicada y que todas las acciones que se toman son para satisfacer intereses políticos, y no se tienen en cuenta todos los actores del transporte.

La industria colombiana insiste en que mientras algunos actores influyentes en el transporte de mercancías en Colombia continúen teniendo las mismas condiciones y obteniendo la misma rentabilidad actual, estos actores no van a apoyar proyectos de ferrocarriles en Colombia, y se continuará con la elección del transporte carretero.

Del análisis de las respuestas y del debate generado en el grupo focal, se obtienen los motivos por los que la industria no está interesada en transportar sus mercancías por ferrocarril. Estos son:

1. Número de transbordos: en las zonas donde opera el ferrocarril, la industria piensa que mientras se minimice el número de transbordos que haga la mercancía, mejor va a ser el transporte en términos de costo y tiempo total.

2. Falta de confianza en el modo: al no ser tan utilizado el ferrocarril, la industria cree que la frecuencia de servicio y la disponibilidad del modo es muy baja, lo que se traduce en sobrecostos y tiempos extras para el transporte de su mercancía.

3. Falta de infraestructura: la industria siente que al no existir la infraestructura necesaria para realizar el cargue y descargue de su mercancía en sus terminales, no es competitivo el ferrocarril.
Del grupo focal se concluyó que las variables que influyen en la industria colombiana para elegir un modo de transporte para transportar sus mercancías son el costo y la frecuencia del servicio del modo en cuestión.

\section{Discusión}

Para el 2014, Colombia contaba con una red ferroviaria de más de $3800 \mathrm{~km}$, de las cuales solo 854 km eran líneas ferroviarias en operación. De estas líneas ferroviarias en operación, el carbón representa el 25,5\% de la mercancía colombiana y se transporta en $350 \mathrm{~km}$ de vías, lo que muestra la gran capacidad que tiene este modo de transporte para mover mercancías en Colombia.

Entre los países que transportaron mayor cantidad de mercancías por ferrocarril en el 2014, se encuentran: China, con 3813 millones t [29]; Estados Unidos, con 1840 millones t [30]; Rusia, con 1375 millones $t$ (datos del 2011) [31] y Australia, con 1298 millones t [32]. Los países de la Unión Europea, por su parte, transportan menos mercancías por ferrocarril que los mencionados anteriormente; los países que más transportaron mercancías por ferrocarril, en el 2014, fueron [33]: Alemania, con 365,0 millones t; Polonia, con 227,8 millones t; Reino Unido, con 108,5 millones t; Austria con 98,3 millones t, y República Checa, con 91,6 millones t. Sumando las cantidades que transportaron todos estos países, la Unión Europea transporta 1734,9 millones de toneladas, y de esta manera se ubica de tercera después de China y Estados Unidos.

El reparto modal entre la Unión Europea y Estados Unidos es similar, donde el transporte de mercancías por ferrocarril en la Unión Europea representó el 18,2\% para el 2013, mientras que por carretera se transportó el $74,9 \%$ de las mercancías y el 6,9\% de las mercancías restantes fueron transportadas por cabotaje [34]. Estados Unidos, en el 2012, transportó por ferrocarril el $18 \%$ de sus mercancías, mientras que por carretera transportó el $70 \%$, y el $12 \%$ restante fue transportado por otros modos [35].

La figura 3 muestra que la industria colombiana no está interesada en transportar sus mercancías por ferrocarril, pues el reparto modal ha sido constante en la última década, salvo la industria carbonera liderada por el Cerrejón que opera 
su línea privada en La Guajira, y Drummond que opera la línea La Loma - Santa Marta.

En la academia y la industria colombiana se expresa la necesidad de que las mercancías sean transportadas por un ferrocarril eficiente para dinamizar la economía nacional. La evidencia reportada $[8,36]$ demuestra que las variables más significativas para transportar mercancías por la industria en una región colombiana son costo y frecuencia de servicio. Luego, modificando los valores de estas dos variables, los autores demostraron que la probabilidad de elección del ferrocarril fluctuaba entre el $67 \%$ y el $93 \%$ para el transporte de mercancías, lo que demuestra que el Gobierno debería tener en cuenta estas dos variables, entre otras, a la hora de reactivar los ferrocarriles en Colombia.

La industria colombiana especula que la única forma para que los proyectos de ferrocarril sean apoyados en Colombia es que todos los actores influyentes en el transporte de mercancías comiencen a tener una mayor visión, buscando el bienestar general sobre el particular, donde prevalezca un pensamiento de nación por encima de intereses individuales.

\section{Conclusiones}

Según los motivos por los que la industria no utiliza el ferrocarril, para que exista un futuro prometedor para el ferrocarril en Colombia es necesario que exista una integración multimodal, y eliminar así la competencia entre los diferentes sistemas de transporte. Para lograr esto es necesario que exista infraestructura complementaria al ferrocarril, como los parques logísticos o puertos secos, y así realizar transbordos de mercancías de una manera rápida, económica y eficiente. Un buen sistema de transporte ferroviario emplearía ferrocarriles para transportar mayor magnitud de mercancías y se utilizarían camiones para distribuir la mercancía en distancias más cortas (esto no podría aplicarse a productos frágiles que deben ser transportados por carretera, sino a aquellos que permitan un transporte multimodal); de esta manera, se ayudaría a movilizar más fácil y rápidamente los productos del interior del país a los puertos y viceversa, entonces el costo de transporte de los productos se reduciría y el mercado colombiano sería más competente.
Es necesario insistir en que una rehabilitación de las líneas inactivas del ferrocarril no es una opción viable, debido a que muchos tramos del ferrocarril están totalmente perdidos y la vía sería obsoleta para los requerimientos actuales. Es entonces necesario construir una nueva red, cambiando trazado y rutas, lo cual conllevaría una inversión inicial mayor que se vería reflejada con los ingresos del futuro. Una renovación absoluta en los ferrocarriles colombianos permitiría el uso de este modo de transporte fundamental para el desarrollo económico y social de Colombia. Tener un moderno sistema de ferrocarril sería valioso para el comercio exterior, ya que abre una brecha de rivalidad en el mercado y trae consigo beneficios como el mejoramiento de la calidad de todos los modos de transporte (ferrocarril, marítimo, aéreo, carretero y fluvial).

Aunque existan estudios que demuestran que el costo y la frecuencia de servicio son variables que influyen en la elección de un modo de transporte para mercancías en por lo menos una región colombiana, es necesario - para trabajos futurosque se investigue a cabalidad cuáles son todas las variables influyentes en la elección del ferrocarril para el transporte de mercancías en Colombia y así tenerlas en cuenta al momento de construir y operar los ferrocarriles para satisfacer estas variables $y$, por consiguiente, a la industria colombiana.

\section{Referencias}

[1] R. Beyer, The Colombian Coffee Industry: Origins and Major Trends, 1740-1940. Minneapolis, Estados Unidos: University of Minnesota, 1947, pp. 80-109.

[2] W. McGreevey, Historia económica de Colombia: 1845. Bogotá, Colombia: Tercer Mundo, 1975.

[3] F. Botero-Herrera, "Estudios sobre la historia económica de Colombia del siglo xix", Revista Lecturas de Economía, n. ${ }^{\circ}$ 9, pp. 175-194, 1982 [en línea]. Disponible en: http://aprendeenlinea.udea.edu.co/ revistas/index.php/lecturasdeeconomia/article/ view/17658

[4] G. Poveda-Ramos, "Nuestra Historia Ferroviaria", Revista Antioqueña de Economía y Desarrollo, n. ${ }^{\circ}$ 21, pp. 6-19, 1986.

[5] M. T. Ramírez, "Railroads and the Colombian Economy", en Econometric Society World Congress 2000 Contributed Papers. Bogotá, Colombia, 2000. 
[6] The World Bank, "Railway, goods transported (million ton-km)", Reporte estadístico, Washington, D.C., Estados Unidos, 2015 [en línea]. Disponible en: http://data.worldbank.org/indicator/IS.RRS. GOOD.MT.K6

[7] L. F. Andrade Moreno, "Cuarta generación de concesiones en Colombia”, Agencia Nacional de Infraestructura, Bogotá, Colombia, Reporte técnico, 2014 [en línea]. Disponible en: https://goo.gl/CRsKnY

[8] J. D. Pineda-Jaramillo, "Modelo de elección de modo de transporte entre carretera y ferrocarril para carga general susceptible a exportación-importación. Aplicación al corredor vial Medellín - puerto de Cartagena", Tesis de Maestría, Facultad de Minas, Universidad Nacional de Colombia, Medellín, Colombia, 2013 [en línea]. Disponible en: http://www. bdigital.unal.edu.co/10618/

[9] Ministerio de Transporte de Colombia, "Transporte en cifras - Estadísticas 2014”, Ministerio de Transporte, Bogotá, Colombia, Reporte estadístico, 2014 [en línea]. Disponible en: https://www.mintransporte.gov.co/descargar.php?idFile $=12621$

[10] The Center for Urban Transportation Research, "Analysis of Freight Movement Mode Choice Factors", The Center for Urban Transportation Research at the University of South Florida, Miami, Estados Unidos, Report for Florida Department of Transportation Rail Planning and Safety, Miami, Estados Unidos, 2011 [en línea]. Disponible en: www.dot. state.fl.us/rail/Publications/Studies/Planning/ModeChoiceFactors.pdf

[11] G. Poveda-Ramos, "El primer Ferrocarril en Colombia”, Revista DYNA, vol. 69, n. ${ }^{\circ} 137$, pp. 61-73, 2002 [en línea]. Disponible en: www.redalyc.org/ pdf/496/49613707.pdf

[12] G. Poveda-Ramos, Historia social de la ciencia en Colombia, Bogotá, Colombia: Tercer Mundo, 1993, pp. 169-197.

[13] G. Duque-Escobar, "El transporte en Colombia y el eje cafetero". En Fundamentos de economía y transportes, parte IV, Manizales, Colombia: Universidad Nacional de Colombia, sede Manizales 2006, p. 41 [en línea]. Disponible en: http://www.bdigital.unal. edu.co/1879/6/04-trans-col-ec.pdf

[14] Departamento Nacional de Planeación, "Plan de rehabilitación de la red férrea nacional”, DNP, Bogotá, Colombia, Estudio técnico, 1991 [en línea]. Disponible en: https://colaboracion.dnp.gov.co/CDT/ Conpes/Econ\%C3\%B3micos/2517.pdf

[15] G. Arias de Greiff, La segunda mula de hierro: historia de los ferrocarriles colombianos a través de sus locomotoras. Bogotá, Colombia: Panamericana, 2006, p. 108.
[16] Corporación Andina del Fomento, Rieles con futuro. Desafíos para los ferrocarriles de América del Sur. Resultados de la reforma. Caracas, Venezuela: Unidad de Publicaciones de la CAF, 2004, p. 279 [en línea]. Disponible en: scioteca.caf.com/bitstream/handle/123456789/423/5.pdf? sequence $=1$ \&isAllowed $=y$

[17] Ministerio de Transporte de Colombia, Resolución Número 135 de 2013 (2013, ene. 25). Por la cual se solicita el registro y anotación a nombre de la $\mathrm{Na}$ ción - Ministerio de Transporte de lote de terreno con matrícula inmobiliaria No. 280-78024 localizado en el municipio de Armenia, departamento de Quindío, que figura en cabeza del Consejo Administrativo de los Ferrocarriles Nacionales y se transfiere a título gratuito al Instituto Nacional de Vías - INVÍAs, [en línea]. Disponible en: https://www.mintransporte. gov.co/descargar.php?idFile $=10171$

[18] Consejo Nacional de Política Económica y Social, Departamento Nacional de Planeación, República de Colombia (2009, abril 1). Documento Conpes 3581. Importancia estratégica del proyecto concesión de la red férrea del Atlántico [en línea]. Disponible en: http://www.ani.gov.co/sites/default/files/documento_conpes_3581_importancia_estrategica_ red_ferrea_del_atlantico.pdf

[19] Departamento Nacional de Planeación, República de Colombia (2010, abril 12). Documento Conpes 3655. Declaratoria de importancia estratégica de la interventoría de la red férrea del Pacífico y modificación al Documento Conpes 3581 de 2009 [en línea]. Disponible en: http://www.ani.gov.co/sites/default/ files/documento_conpes_3655_red_ferrea_pacifico.pdf

[20] Cámara Colombiana de la Infraestructura, "Seguimiento a Proyectos de Infraestructura: sistema ferreo nacional”, Bogotá, Colombia, Informe Dirección Técnica 2012 [en línea]. Disponible en: http://www.infraestructura.org.co/seguimientoproyectos/Informe $\% 20$ ferrocarriles.pdf

[21] Incoplan S. A. Ingeniería, Consultoría y Planeación, "Estudio técnico sectorial "Infraestructura de transporte multimodal y de logísticas integradas para el desarrollo de la industria minera en Colombia, con énfasis en puertos", Incoplan, Bogotá, Colombia, Informe final, 2011 [en línea]. Disponible en: www. simco.gov.co/LinkClick.aspx?fileticket=0CBLMjO $\mathrm{Nx} 3 \mathrm{M}=$ \&tabid=282

[22] J. Kohon, J. Champin, M. Rodríguez y R. Cortés, Desafíos del transporte ferroviario de carga en Colombia. Bogotá, Colombia: Banco Interamericano de Desarrollo - BID, 2016 [en línea]. Disponible en: https:// publications.iadb.org/handle/11319/7558?localeattribute $=$ en 
[23] Ministerio de Transporte de Colombia, "Transporte en cifras. Versión 2010”. Bogotá, Colombia, Documento estadístico del sector transporte, 2010 [en línea]. Disponible en: https://www.mintransporte. gov.co/descargar.php?idFile $=53$

[24] D. Zinoviev, Railway Gauge Width, 2016 [en línea]. Disponible en: http://parovoz.com/spravka/gaugesen.php

[25] F. González, "La pugna por el carbón de El Cerrejón en el siglo XIx", El Heraldo, 27, octubre 2012. [en línea]. Disponible en: http://revistas.elheraldo.co/ latitud/la-pugna-por-el-carbon-de-el-cerrejon-enel-siglo-xix-87176

[26] Drummond Ltd., DrummondCO, Santa Marta, Colombia, 2016 [en línea]. Disponible en: http://www.drummondltd.com/our-operations/ mines/?lang=en

[27] D. L. Morgan y R. A. Krueger, When to use focus groups and why, in D. L. Morgan (Ed.), Successful focus groups: Advancing the state of the art. California, Estados Unidos: SAge Publishing, 1993, pp. 3-19.

[28] R. Breen, "A Practical Guide to Focus-Group Research", Journal of Geography in Higher Education, vol. 30, n. ${ }^{\circ} 3$, pp. 463-475, 2006 [en línea]. Disponible en: http://www.tandfonline.com/doi/ abs/10.1080/03098260600927575

[29] National Railway Administrarion of the People's Republic of China, "Ferrocarril 2014", Pekín, China, Reporte estadístico, 2014 [en línea]. Disponible en: http://www.nra.gov.cn/fwyd/zlzx/hytj/201504/ t20150427_13281.htm
[30] Association of American Railroads, "Class 1 Railroad statistics", Washington, Estados Unidos, 3, mayo 2016 [en línea]. Disponible en: https://www. aar.org/Documents/Railroad-Statistics.pdf

[31] Ministerio de Comunicaciones de Rusia, "EMIss Estadísticas del Gobierno”, Moscú, Rusia, Reporte estadístico, 2016 [en línea]. Disponible en: https:// fedstat.ru/indicator/31314.do

[32] Australian Government, Bureau of Infrastructure, Transport and Regional Economics and Australasian Railway Association", "Rail. Trainline 3", Canberra, Australia, Reporte estadístico, 2015 [en línea]. Disponible en: http://bitre.gov.au/publications/2015/files/trainline_003.pdf

[33] European Union, "Eurostat", [en línea]. Disponible en: http://ec.europa.eu/eurostat/web/transport/ data/database

[34] European Union, "Freight transport statistics" [en línea]. Disponible en: http://ec.europa.eu/eurostat/ statistics-explained/index.php/Freight_transport_ statistics

[35] U. s. Department of Transportation, "American Industry Shipped 11.7 Billion Tons of Goods in 2012", Bureau of transportation statistics, 10, dic. 2013 [en línea]. Disponible en: http://www.rita.dot. gov/bts/press_releases/bts055_13

[36] J. D. Pineda Jaramillo e I. Sarmiento Ordosgoitia, "Variables influyentes en la elección entre carretera y ferrocarril para carga general de comercio exterior", Ingeniería Solidaria, vol. 10, no. 17, pp. 29-37, 2014. 
